\title{
Plastic and Reconstructive Surgery
}

M POOLE

M BRIGGS

J RAYNE

H CHENG

\section{CRANIOFACIAL SURGERY}

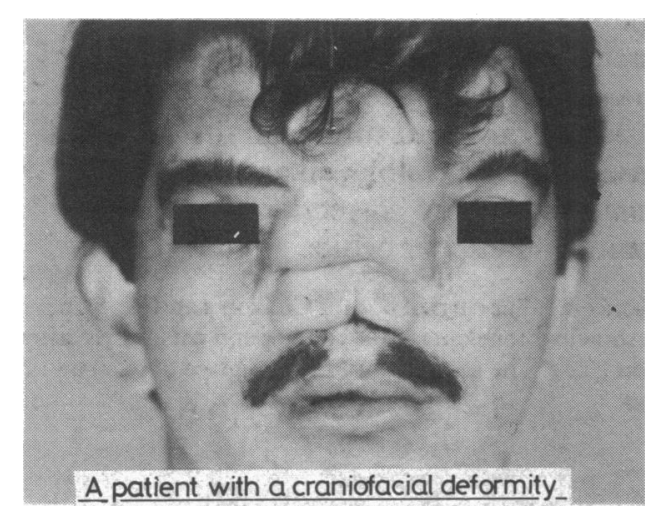

\section{Congenital anomalies}
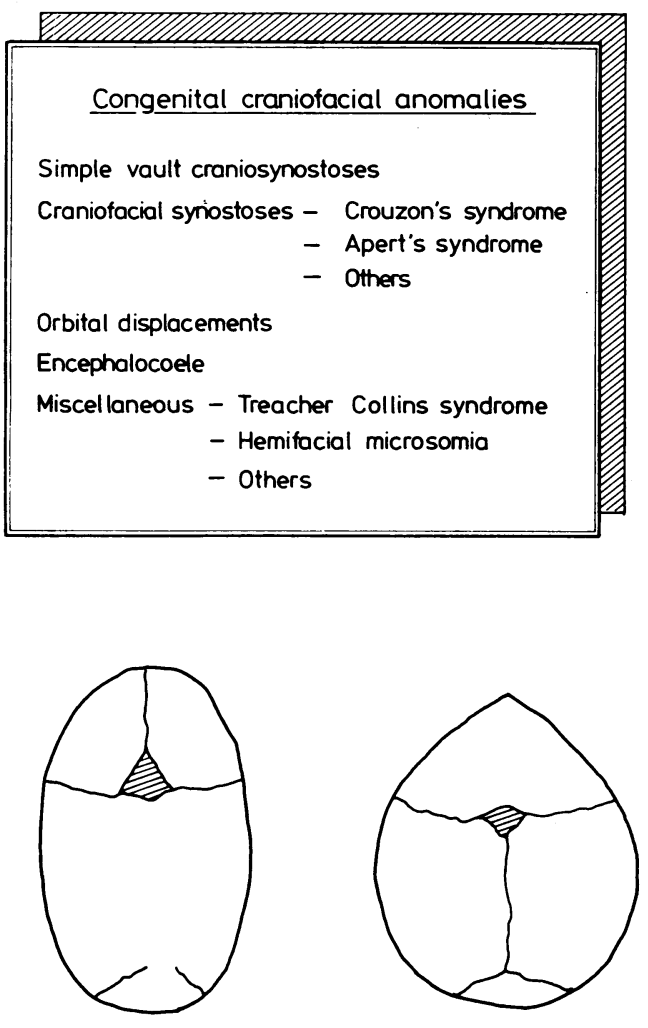

Scaphocephaly (sagittal suture synostosis) (metopic suture synostosis)
Craniofacial surgery is concerned with surgical correction of problems affecting the skull base, orbits, and upper face. These anatomical areas, in particular the skull base, were until recently a kind of "no man's land" that did not fall within the province of any particular surgical specialty. In recent years multidisciplinary teams have been formed to deal with problems in this difficult area. Because these problems are not common and management is difficult craniofacial surgery is performed in only a few centres with surgical teams treating sufficient numbers of patients to maintain the required level of skill. The conditions treated in this way fall into three main groups: congenital anomalies, tumours, and trauma.

The craniosynostoses are a group of disorders in which one or more of the sutural joins between the cranial bones becomes prematurely ossified, impairing the growth of the skull that takes place in a direction at right angles to the suture. This may lead to a deformity of the cranium (and eventually to deformity of the facial skeleton in some types) and, in some children, to mildly raised intracranial pressure, which may be harmful. Many cases of simple craniosynostosis do not require surgery and are managed expectantly, but in those where surgery is necessary it is usually done fairly early in infancy to obtain the best results in terms of subsequent growth. The surgical procedure in some of these conditions needs to be carried into the anterior skull base and upper face.

Iypes of craniosynostosis
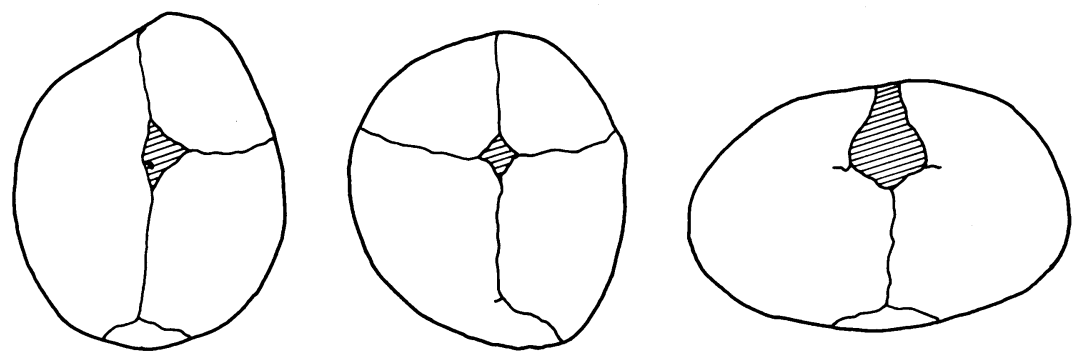

Plagiocephaly (unilateral coronal 


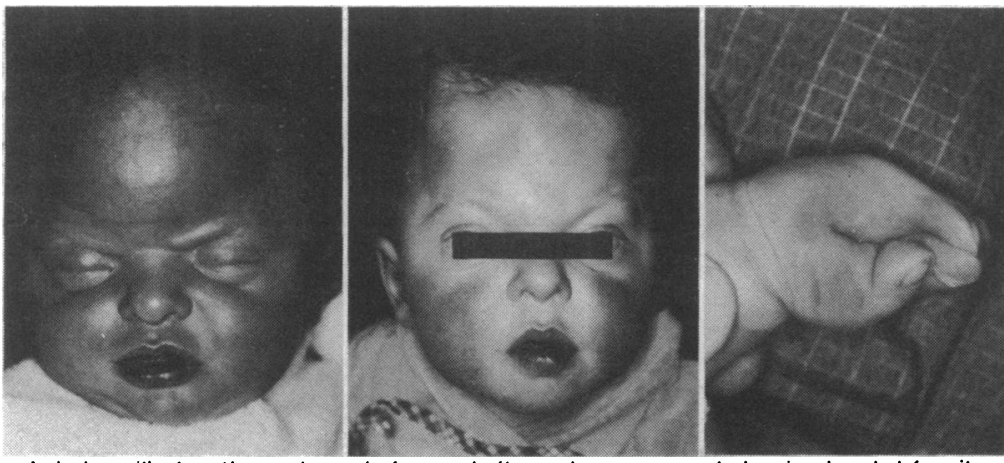

A baby with Apert's syndrome betore and atter early surgery and showing hand deformity
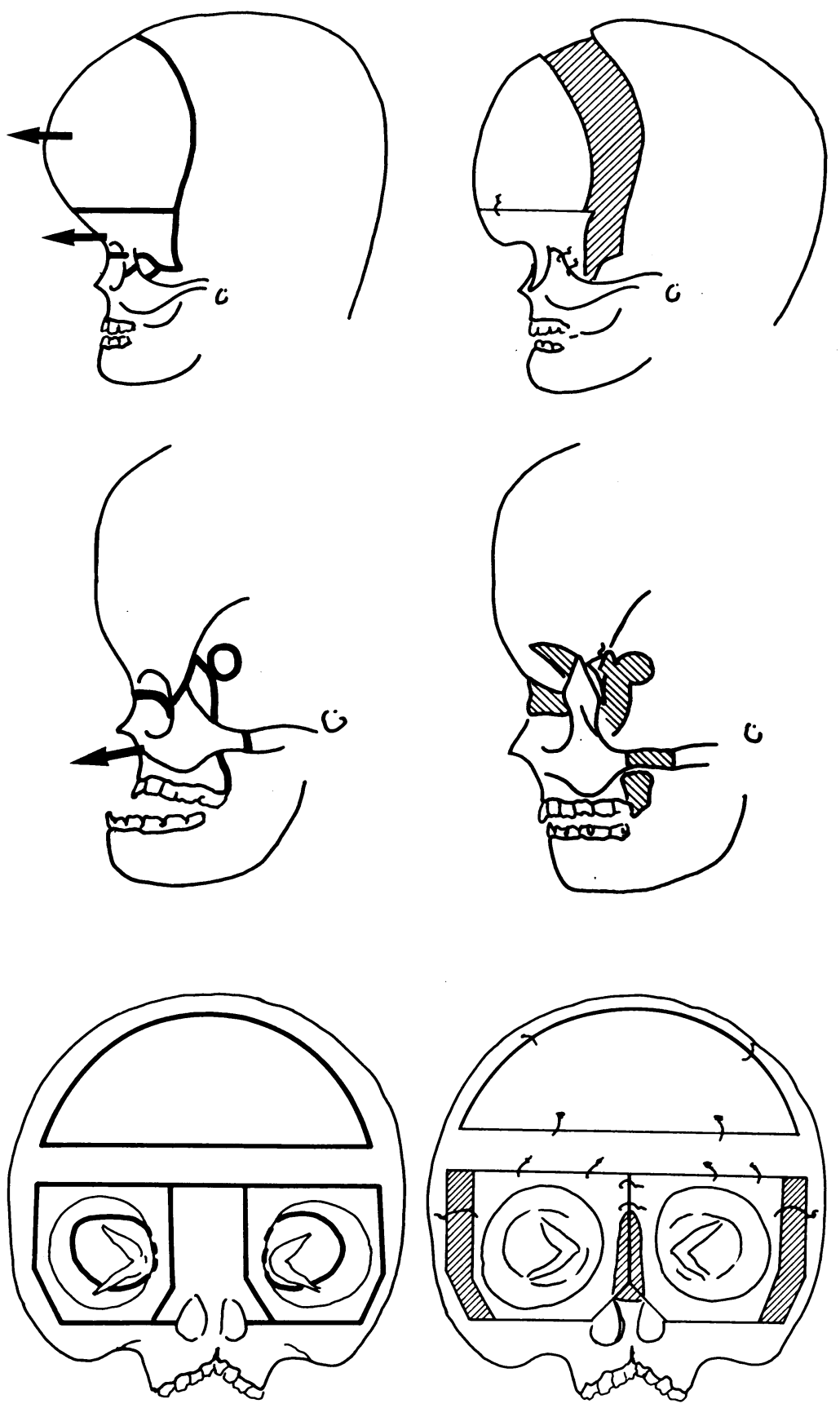

Correction of hypertolerism showing bone cuts — and bone grafts 1010
The craniofacial synostoses have in common the premature fusion of the cranial vault sutures together with developmental failure of the growth areas of the skull base and upper face. The deformities that may result are therefore more complex and severe, and surgical correction is more often necessary. Several syndromes fall into this group, the most common being Crouzon's syndrome and Apert's syndrome. Apert's syndrome is sometimes included in a batch of disorders called collectively acrocephalosyndactyly; syndactyly of hands and feet also occurs. These may be genetically transmitted.

Surgery in these conditions is directed at relieving raised intracranial pressure and allowing as far as possible for normal growth and development of brain and cranium in infancy. These procedures usually require removal of bone from the sites of the fused sutures in the vault and cranial base where accessible, enlargement of the intracranial volume, and correction of asymmetry in the cranium and upper orbits.

Frontal advancement as carried out for Conzon's and Apert's syndromes showing bone cuts (-) and open area left free after advancement ( the forehead.

Later surgery, generally in early childhood, is directed at correcting any facial deformity, which may be gross. For example in some children with Crouzon's and Apert's syndromes a form of "dish face" develops, with proptosed eyes, resulting from limited growth of the mid-face. This can be vastly improved by a procedure to advance the whole mid-facial skeleton. All this surgery is done as far as possible through an approach via a coronal scalp incision to avoid scars on the facial skin.

Le Fort III mid-facial advancement showing bone cuts ( and bone grafts $(\mathrm{Z})$.

Orbital problems-The whole of one or both bony orbits may be displaced in any direction as part of a congenital anomaly that may be associated with a facial cleft or zone of hypoplasia, an encephalocoele, or as part of craniofacial synostosis. These abnormalities produce a bizarre facial appearance and, often, visual problems. The commonest anomaly of this type is hypertelorism, in which the orbits are widely separated with an abnormal nose in between. Craniofacial surgical techniques have been developed in which the parts of the orbits containing the eyeballs may be safely moved into more normal relation. This can be done in early childhood. There may be extensive soft tissue deformities in addition to skeletal ones in some of these conditions; these often require further "tidy up" procedures. The ultimate result depends largely on these soft tissue problems and may be short of ideal. Squint surgery is often necessary at some stage in the management of these children. 


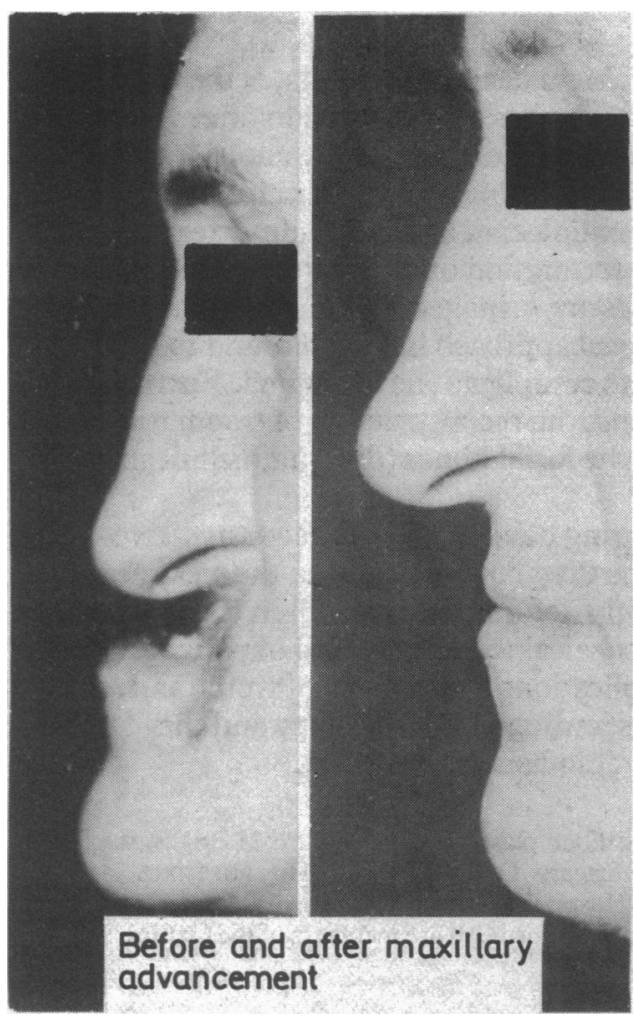

Miscellaneous deformities affecting the head and face are often amenable to surgical correction. Not all of these require combined neurosurgical and plastic surgical procedures, but their management has been made much easier, safer, and more effective as a result of the multidisciplinary approach and new techniques used in craniofacial surgery. Examples of this are the Treacher Collins syndrome and hemifacial microsomia. The Treacher Collins syndrome may be extremely deforming and associated with complex squints as well as problems of dental occlusion. Hemifacial microsomia, with changing facial asymmetry as growth proceeds, similarly has several facets to its management. Many of the children with monstrous abnormalities have, contrary to popular belief, normal intelligence. Consequently, surgical correction to as near normal as possible is worth while and should be done as early as possible. Some patients with these deformities develop abnormalities of jaw relation with consequent effects on facial contour and dental occlusion. These problems are common in patients with clefts of the lip and palate but may be found in others without clefts and are almost always present in those with Apert's and Crouzon's syndromes. Orthognathic surgery as practised by oral and maxillofacial surgeons may thus form part of the multidisciplinary approach and, because of the sensitivity of the mouth to very minor disturbances of dental occlusion, requires careful planning. Complex ancillary technical help is necessary to contribute to positioning and external fixation of the surgical fractures. This work has made an appreciable impact on the management of these patients, with often spectacular improvement in appearance.

\section{Tumours and tumour like conditions}

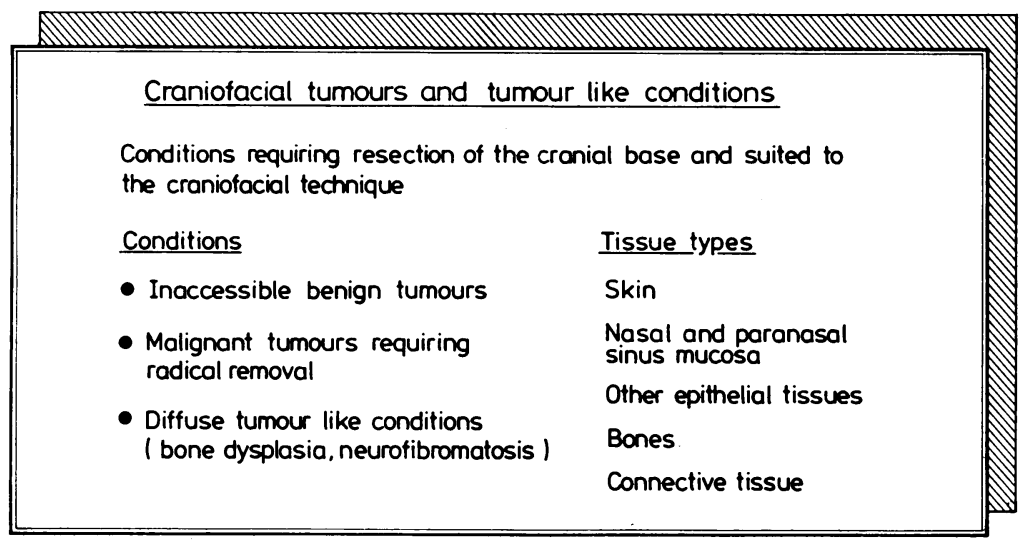

The table shows the tumours that can be dealt with by craniofacial surgical techniques. Patients with tumours in the craniofacial region need careful assessment, with computed tomographic scanning in particular, to define accurately the limits of tumour spread and plan the required resection and reconstruction. Not all tumours in this region require surgery, but it can be used if other methods of treatment are not the most appropriate. The aim in tumours for which surgery is indicated is total removal, and such a resection may include the bone of the skull base if that is affected, the dura mater, and even, if necessary, some of the brain.

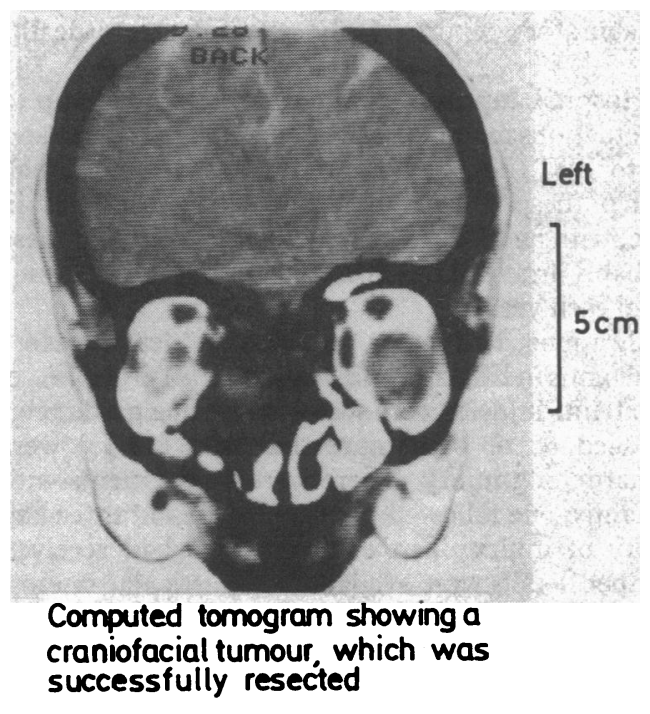

The reconstructive aspects of this surgery are considerable and require repair of the dura, the cranial base, the lining tissues of the nasal roof, and other adjacent structures to produce a well protected cranial cavity and as good an appearance as possible. Success in dealing with craniofacial tumours depends largely on the histological type of growth and on its extent. Appreciable numbers of patients with these until recently inoperable tumours can be salvaged by this surgical approach when carefully selected.

A group of tumour like conditions, which behave like tumours, are also treated by craniofacial resection and repair. The bone dysplasias, which may affect the craniofacial skeleton, in particular fibrous dysplasia, may require surgery. In general, the only way to arrest surgically the progression of the gross bony enlargement that can develop in this condition is to resect (completely if at all possible) the affected area of bone and reconstruct it with bone grafts from unaffected bones. Such procedures are possible. Another tumour like disorder is neurofibromatosis, affecting the region around the orbit, a most difficult condition to treat but one that has been dealt with in craniofacial centres with some degree of success. 


\section{Craniofacial trauma}

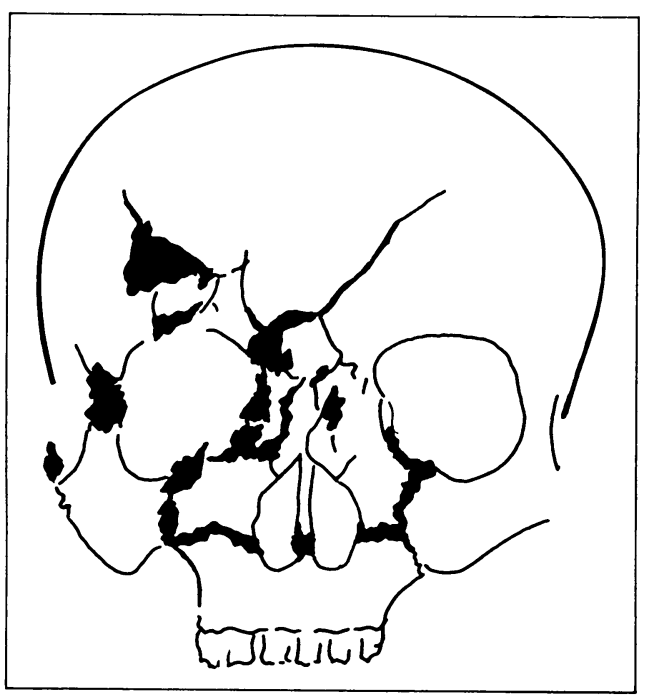

Craniofacial trauma, still a problem despite seat belts, is the third main area where craniofacial surgical techniques have much to offer. Most of this kind of trauma, including extensive damage to the anterior cranial base, frontal sinus, orbits, and jaws, requires a multidisciplinary approach with a neurosurgical, plastic, maxillofacial, and ophthalmic team contributing as necessary to the restoration of satisfactory function and appearance. Generally these cases are managed in regional neurosurgical centres. The combined craniofacial approach has been found to reduce the time in the acute hospital and late complications of trauma. Further surgical procedures-for instance, the reconstruction of traumatically displaced orbits or malunion of the facial bones (difficult technically) - are seldom required.

Craniofacial surgery is an exciting development in reconstructive surgery and has already made startling contributions to the treatment of some of the most difficult and ugly deformities encountered. The future will see refinements of existing surgical techniques and of patient management, probably new applications for this sort of work, and, with further research, a better understanding of the aetiology and the complexities of the congenital craniofacial deformities.

Mr Michael D Poole, MB, FRCs, is consultant plastic surgeon and Mr M Briggs, MSC, FRCS, is consultant neurosurgeon, Radcliffe Infirmary, Oxford; J Rayne, FDS, DORTHRCS, is consultant oral surgeon, John Radcliffe Hospital, Oxford; and H Cheng, FRCS, is consultant ophthalmic surgeon, Oxford Eye Hospital, Oxford. All are members of the Oxford Craniofacial Unit.

\section{Communicable Diseases}

\section{Tetanus surveillance: England and Wales, 1981-3}

Three routine sources of national data on tetanus-namely, statutory notifications, laboratory reports, and death certificationwere analysed for the three years 1981-3. Data from the Hospital Inpatient Enquiry from 1977-81 were also considered. A previous review of these data covered the period $1930-79,{ }^{1}$ and a subsequent surveillance report the year $1980 .^{2}$ Since 1981 a special inquiry has been made by the Communicable Disease Surveillance Centre about each notified case of tetanus, which has made it possible to integrate notifications with laboratory reports and certified deaths and also to collect more information about the circumstances associated with the disease.

\section{Results}

There were 15 notifications in 1981, 12 in 1982, and only six in 1983 , the lowest annual number recorded since notification began in 1969 (figure). In the 15 years since then notifications have fallen progressively. Altogether in this period there were 264 notifications, 142 men and 122 women, a male:female ratio of $1 \cdot 2$. This ratio did not change over the 15 years. The ratio of notification rates over the same period, however, varied with age; the male:female ratio in the age group 0-44 years was $2 \cdot 8$, but in those over 45 it was $0 \cdot 7$.

The age distribution of notifications changed during the 15 years; between 1969 and $197322 \%$ of patients were aged $0-14$ years but by 1974-8, this had fallen to $5 \%$ and even lower, to $3 \%$, between 1979 and 1983. Indeed, tetanus in children almost disappeared with only two notified cases in the three years 1981-3.

There were 20 laboratory reports of tetanus between 1981 and
1983. The decline in laboratory reports over the three years was similar to the decline in notifications; there were 11 reports in 1981, six in 1982, and three in 1983. Of the 20 cases reported by laboratories only six were included among the 33 cases notified in this period.

Eight deaths were attributed to tetanus in 1981-3 and in six others tetanus was a contributory factor. Of the 14 deaths eight were in 1981 , four in 1982, and two in 1983, the lowest number ever recorded. The data followed the same downward trend observed in notifications and laboratory reports. Only seven of the 14 deaths were also notified.

Data from the Hospital Inpatient Enquiry were available up to 1981. The wide fluctuations in the estimated hospital discharges and deaths from 1977 to 1981 (figure) were probably due to the small sample size; in each of these five years there were five cases or less in the original sample, with an average of three a year. Based on these data there was probably an average of about 30 patients with tetanus admitted to hospital each year.

Integrating notifications, laboratory reports, and deaths gave a total of 52 cases of tetanus in England and Wales in 1981-3 (table).

In 21 of the 52 patients injuries were sustained during gardening; indeed, in those aged 65 or over more than half (15/26) were associated with a gardening injury. There were four patients with "occupational" tetanus; one followed a sports injury and three had no apparent history of injury. Ten other patients had received injuries but no further details were available. Of particular interest were two patients with tetanus after cholecystectomy in the Northern region for which there was no obvious explanation. ${ }^{3}$ No information was available in the remaining cases. It proved 